\title{
A SUFFICIENT CONDITION FOR THE SECOND DERIVED FACTOR GROUP TO BE FINITE
}

\author{
by J. R. HOWSE* \\ (Received 10th August, 1985)
}

\section{Introduction}

This paper concerns an application of an algorithm for the second derived factor group as described by Howse and Johnson in [3]. This algorithm has as its basis the Fox derivative (see [1]), a mapping from the free group $F$ to the group-ring $\mathbb{Z} F$, defined as follows: let $X$ be a set of generators of a group $G$, and let $w=y_{1} \ldots y_{k}$ with each $y_{i} \in X^{ \pm 1}$. Then the Fox derivative of the word $w$ with respect to any generator $x \in X$ is defined to be

$$
\frac{\partial w}{\partial x}=\sum_{i=1}^{k} a_{i}, \quad \text { where } a_{i}=\left\{\begin{array}{lll}
y_{1} \ldots y_{i-1}, & \text { when } y_{i}=x \\
-y_{1} \ldots y_{i}, & \text { when } y_{i}=x^{-1} \\
0, & \text { when } y_{i} \neq x^{ \pm 1}
\end{array}\right.
$$

Let $\phi: F \rightarrow G$ (and also $\phi: \mathbb{Z} F \rightarrow \mathbb{Z} G$, etc.) and $\psi: G \rightarrow G / G^{\prime}$ (and also $\psi: \mathbb{Z} G \rightarrow \mathbb{Z}\left(G / G^{\prime}\right)$, etc.). The Jacobian $J=\partial R / \partial X$ of the presentation $G=\langle X \mid R\rangle$ is the $|R| \times|X|$ matrix whose $(i, j)$ entry is $\partial r_{i} / \partial x_{j}$. Let $G / G^{\prime}=\left\{z_{1}, \ldots, z_{n}\right\}$ and $A$ be a matrix over $\mathbb{Z}\left(G / G^{\prime}\right)$. Any entry $\gamma \in \mathbb{Z}\left(G / G^{\prime}\right)$ of $A$ is of the form $\gamma=\sum_{i=1}^{n} \alpha_{i} z_{i}$ and thus defines an $n$-tuple $\left(\alpha_{1}, \ldots, \alpha_{n}\right)$. The $n$-tuple corresponding to $z_{j} \gamma(1 \leqq j \leqq n)$ is a rearrangement of this, and we let $m(\gamma)$ denote the $n \times n$ matrix having this as its $j$ th row. Let $m(A)$ denote the matrix of integers obtained by applying $m$ to each entry of $A$. Then the integer matrix $M=m(\psi \phi(J))$ is a relation matrix for the group $G^{\prime} / G^{\prime \prime} \oplus Z^{\oplus(n-1)}$. The invariant factors of $G^{\prime} / G^{\prime \prime}$ can be computed from $M$ by diagonalisation.

The proof of this algorithm together with examples illustrating it and applications of it can be found in [2] and [3].

This paper applies the algorithm to 2-generator groups with finite derived factor groups. The main result obtained is that the second derived factor group is finite if the determinant of the matrix, with the above notation, $A_{i j}=m\left(\psi \phi\left(\partial r_{i} / \partial x_{j}\right)\right)$ for some $r_{i} \in R$ and $x_{j} \in X$ of the group presentation $G=\langle X \mid R\rangle(|X|=2)$, is non-zero. This result is then applied to groups with cyclic derived factor group and which have a presentation which 
contains at least one relator having a small number of syllables; in which case much more explicit conditions for the second derived factor group to be finite are determined.

In the application of the algorithm the integer matrix $m(\psi \phi(\partial r / \partial x))$ can be represented by the "polynomial" $\psi \phi(\partial r / \partial x)$, e.g. if $r=x^{3}$, then $\psi \phi(\partial r / \partial x)=1+x+x^{2}$ and this can represent the integer matrix

$$
\left(\begin{array}{lll}
1 & 1 & 1 \\
1 & 1 & 1 \\
1 & 1 & 1
\end{array}\right)
$$

(assuming that $G / G^{\prime} \cong \mathbb{Z}_{3}$ ). Moreover the integer relation matrix $m(\psi \phi(J)$ ) can be represented by the "polynomial" matrix $\psi \phi(J)$. Indeed row and column operations can be performed on this "polynomial" matrix.

\section{The main theorem}

Consider the 2-generator group $G=\left\langle x, y \mid r_{1}, \ldots, r_{q}\right\rangle$, where $2 \leqq q<\infty$, with finite derived factor group and $\left|G: G^{\prime}\right|=n$. For $i=1, \ldots, q$, let $r_{i}=x^{a_{i 1}} y^{b_{i 1}} \ldots x^{a_{i k}} y^{b_{i k_{i}}}$ where $a_{i h} \neq 0$ and $b_{i h} \neq 0$ for $h=1, \ldots, k_{i}$, if $k_{i}>1$.

Let $\sum_{h=1}^{k_{i}} a_{i h}=a_{i}$ and $\sum_{h=1}^{k_{i}} b_{i h}=b_{i}$. Let $G / G^{\prime}=\left\{z_{1}, \ldots, z_{n}\right\}$. Then the Fox derivatives of the relator $r_{i}$ with respect to the generators $x$ and $y$, modulo $G^{\prime}$, are of the form

$$
\begin{aligned}
& \frac{\partial r_{i}}{\partial x} \equiv \alpha_{i 1} z_{1}+\cdots+\alpha_{i n} z_{n} \quad\left(\bmod G^{\prime}\right), \\
& \frac{\partial r_{i}}{\partial y} \equiv \beta_{i 1} z_{1}+\cdots+\beta_{i n} z_{n} \quad\left(\bmod G^{\prime}\right)
\end{aligned}
$$

Lemma 1. $\alpha_{i 1}+\cdots+\alpha_{i n}=a_{i}$ and $b_{i 1}+\cdots+b_{i n}=b_{i}$.

The proof is obvious from the definition of Fox derivatives.

The matrix (given in polynomial form)

$$
J=\left(\begin{array}{cc}
\alpha_{11} z_{1}+\cdots+\alpha_{1 n} z_{n} & \beta_{11} z_{1}+\cdots+\beta_{1 n} z_{n} \\
\vdots & \vdots \\
\alpha_{q 1} z_{1}+\cdots+\alpha_{q n} z_{n} & \beta_{q 1} z_{1}+\cdots+\beta_{q n} z_{n}
\end{array}\right)
$$

is a relation matrix for $G^{\prime} / G^{\prime \prime} \oplus \mathbb{Z}^{\oplus(n-1)} \cdot m(J)$ is a $q n \times 2 n$ matrix, thus $\left|G^{\prime}: G^{\prime \prime}\right|$ is equal to the h.c.f. of the determinants of all $(n+1)$-rowed minors of $m(J)$ when finite, and is infinite when all these are zero.

Consider the $2 n \times 2 n$ "submatrix" of $J$

$$
\left(\begin{array}{ll}
\alpha_{i 1} z_{1}+\cdots+\alpha_{i n} z_{n} & \beta_{i 1} z_{1}+\cdots+\beta_{i n} z_{n} \\
\alpha_{j 1} z_{1}+\cdots+\alpha_{j n} z_{n} & \beta_{j 1} z_{1}+\cdots+\beta_{j n} z_{n}
\end{array}\right)=K_{i j} \text { (say) }
$$


where $i \neq j$. In integer form we will write this matrix as

$$
m\left(K_{i j}\right)=\left(\begin{array}{ll}
m\left(\alpha_{i 1}, \ldots, \alpha_{i n}\right. & m\left(\beta_{i 1}, \ldots, \beta_{i n}\right. \\
m\left(\alpha_{j 1}, \ldots, \alpha_{j n}\right. & m\left(\beta_{j 1}, \ldots, \beta_{j n}\right.
\end{array}\right) .
$$

Replace row $n+1$ by the sum of the last $n$ rows, and column $n+1$ by the sum of the last $n$ columns and then consider the first $n+1$ rows and $n+1$ columns of the resulting matrix to get (using Lemma 1)

$$
\left(\begin{array}{cccc}
m\left(\alpha_{i 1}, \ldots, \alpha_{i n}\right) & & b_{i} \\
& & \vdots \\
a_{j} & \ldots & a_{j} & n b_{j}
\end{array}\right)=M_{i j} \text { (say). }
$$

Now $M_{i j}$ is an $(n+1) \times(n+1)$ matrix (while not an $(n+1)$-rowed minor of $J, M_{i j}$ was produced from $J$ by matrix operation), thus we have

$$
\left|G^{\prime}: G^{\prime \prime}\right||| \operatorname{det} M_{i j} \mid
$$

Lemma 2. If $a_{i} \neq 0$, then

$$
\left|\operatorname{det} M_{i j}\right|=n\left|a_{i} b_{j}-a_{j} b_{i}\right|\left|\operatorname{det} A_{i}\right| /\left|a_{i}\right|
$$

where $A_{i}=m\left(\alpha_{i 1}, \ldots, \alpha_{i n}\right)$.

Proof.

$$
\begin{aligned}
& \left|\operatorname{det} M_{i j}\right|=\left|\operatorname{det}\left(\begin{array}{cccc}
A_{i} & & & b_{i} \\
& & & \vdots \\
& & & b_{i} \\
a_{j} & \ldots & a_{j} & n b_{j i}
\end{array}\right)\right|
\end{aligned}
$$

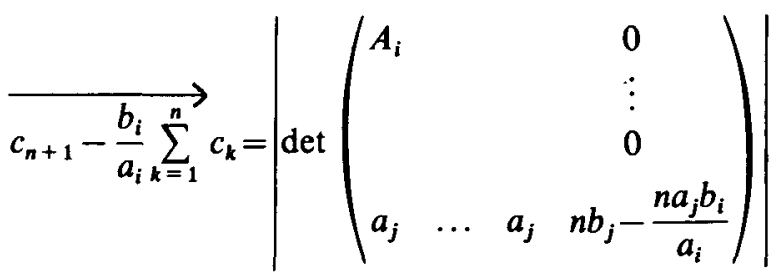

$$
\begin{aligned}
& =n\left|\frac{a_{i} b_{j}-a_{j} b_{i}}{a_{i}}\right|\left|\operatorname{det} A_{i}\right| \text {, as required. }
\end{aligned}
$$

Lemma 3. If $a_{i} \neq 0$, then $a_{i} b_{j}-a_{j} b_{i} \neq 0$ for some $j$. 
Proof. From the original definition of $G$, we have

$$
\left|G: G^{\prime}\right|=\text { h.c.f. }\left(a_{i} b_{j}-a_{j} b_{i} ; i=1, \ldots, q, j=1, \ldots, q\right) \text {. }
$$

We will assume throughout this proof that $a_{i} \neq 0$. If $b_{i}=0$, then there exists $j$ such that $b_{j} \neq 0$ from (2), because $G / G^{\prime}$ is finite. So if $b_{i}=0$, then $a_{i} b_{j}-a_{j} b_{i} \neq 0$ for some $j$. Now consider the case $b_{i} \neq 0$. Assume, for a contradiction, that $a_{i} b_{j}-a_{j} b_{i}=0$ for all $j$. Thus $a_{j}=a_{i} b_{j} / b_{i}$ for all $j$. So for all $j, k$ we have

$$
a_{k} b_{j}-a_{j} b_{k}=\frac{a_{i} b_{k}}{b_{i}} b_{j}-\frac{a_{i} b_{j}}{b_{i}} b_{k}=0
$$

contradicting (2), because $G / G^{\prime}$ is finite. Thus $a_{i} b_{j}-a_{j} b_{i} \neq 0$ for some $j$.

We can now state a sufficient condition for $\left|G^{\prime}: G^{\prime \prime}\right|$ to be finite.

Theorem 1. Let $G$ be a 2-generator group with $G / G^{\prime}$ finite. Let $A_{i}=m\left(\psi \phi\left(\partial r_{i} / \partial x_{j}\right)\right)$ for a given generator $x_{j}$. If $\operatorname{det} A_{i} \neq 0$ for some $i$, then $G^{\prime} / G^{\prime \prime}$ is finite.

Proof. If $a_{i}=0$, then $\operatorname{det} A_{i}=0$, because each row-sum $=a_{i}=0$, by Lemma 1 ; however, there exists $i$ so that $a_{i} \neq 0$, by the hypothesis that $\operatorname{det} A_{i} \neq 0$. Assume that $a_{i} \neq 0$. By Lemma $3, a_{i} b_{j}-a_{j} b_{i} \neq 0$ for some $j$; so if $\operatorname{det} A_{i} \neq 0$, then $\operatorname{det} M_{i j} \neq 0$ by Lemma 2. Thus by (1), $G^{\prime} / G^{\prime \prime}$ is finite.

\section{Groups with cyclic derived factor group}

When the derived factor group is cyclic, the matrix $A_{i}$ of Theorem 1 is circulant. A formula for the determinant of a circulant matrix is given in Lemma 4 below. From this formula conditions can be found such that the determinant is not zero, giving further conditions for the second derived factor group to be finite.

Lemma 4. Let $C=C\left(\alpha_{1}, \alpha_{2}, \ldots, \alpha_{n}\right)$ be a circulant matrix, and $\omega$ be a primitive $n$th root of unity. Then

$$
\operatorname{det} C=\prod_{i=1}^{n} \sum_{j=1}^{n} \alpha_{j} \omega^{i(j-1)}
$$

The proof of this lemma can be found in [4].

The following theorems are concerned with groups with cyclic derived factor group. We will consider groups having a presentation which contains at least one relator having a small number of syllables, i.e. a relator of the form $r=x^{a} y^{b}$, or of the form $r=x^{a_{1}} y^{b_{1}} x^{a_{2}} y^{b_{2}}$.

Let $G=\langle x, y \mid R\rangle$, where $R$ is a finite set of relators, with $G / G^{\prime}=\left\langle z \mid z^{n}\right\rangle$, where $x=z^{m_{1}}\left(\bmod G^{\prime}\right)$ and $y \equiv z^{m_{2}}\left(\bmod G^{\prime}\right)$, where $0<m_{1} \leqq n-1$ and $0<m_{2} \leqq n-1$. (It should 
be noted that $0<m_{1}, m_{2}$ is an extra assumption, but the case $x \equiv 1\left(\bmod G^{\prime}\right)$, i.e. $m_{1}=0$, is considered in [2]. Moreover, either $m_{1}$ or $m_{2}$ is not zero, unless $G=G^{\prime}=G^{\prime \prime}$.)

Let $M_{j}=\left\{s ; s \mid m_{j}\right\}(j=1,2)$ the set of all divisors of $m_{j}$.

Theorem 2. Let $G$ be as just stated. Let $r=x^{a} y^{b}$, where $a \neq 0, r \in R$. If $\left(a m_{1}, n\right) \in M_{1}$, then $G^{\prime} / G^{\prime \prime}$ is finite.

Proof. Without loss of generality we can assume that $a>0$ (if $a<0$, then the relator $r=x^{a} y^{b}$ can be rewritten as $y^{-b} x^{-a}$ and then as $\left.x^{-a} y^{-b}\right)$. Now

$$
\begin{aligned}
\frac{\partial r}{\partial x}=\frac{\partial x^{a}}{\partial x} & =1+x+\cdots+x^{a-1} \\
& \equiv 1+z^{m_{1}}+z^{2 m_{1}}+\cdots+z^{(a-1) m_{1}} \quad\left(\bmod G^{\prime}\right)
\end{aligned}
$$

Let $f(z)=1+z^{m_{1}}+z^{2 m_{1}}+\cdots+z^{(a-1) m_{1}}$. (Recall that $z^{n}=1$ ). Then, by Lemma $4, \operatorname{det} A=$ $\prod_{z^{n}=1} f(z)$ (where $A$ represents, in this case, the matrix $A_{i}$ of Theorem 1).

If det $A=0$, then $f(w)=0$ for some $n$th root $w$ of unity, and if $\operatorname{det} A \neq 0$, then $G^{\prime} / G^{\prime \prime}$ is finite by Theorem 1 . Let $\left(a m_{1}, n\right) \in M_{1}$, and, for a contradiction, assume that $f(w)=0$ where $w$ is an $n$th root of unity. Then

$$
\left(1-w^{m_{1}}\right) f(w)=0 \Rightarrow 1-z^{a m_{1}}=0 \Rightarrow z^{a m_{1}}=1 .
$$

Thus $w^{\left(a m_{1}, n\right)}=1$ and hence $w^{m_{1}}=1$, because $\left(a m_{1}, n\right) \in M_{1}$ and so $\left(a m_{1}, n\right)$ divides $m_{1}$. So

$$
f(w)=1+w^{m_{1}}+\cdots+w^{(a-1) m_{1}}=a \neq 0
$$

the required contradiction. So $\operatorname{det} A \neq 0$ and $G^{\prime} / G^{\prime \prime}$ is finite by Theorem 1 .

Theorem 3. Let $G$ be as defined above. Let $r=x^{a_{1}} y^{b_{1}} x^{a_{2}} y^{b_{2}}$, where $r \in R$ and $a_{1}+a_{2} \neq 0$.

(i) Let $n$ be odd. If $\left(a_{1} m_{1}, a_{2} m_{1}, n\right) \in M_{1}$ and $\left(b_{1} m_{2}, b_{2} m_{2}, n\right) \in M_{1}$, then $G^{\prime} / G^{\prime \prime}$ is finite.

(ii) Let $n$ be even. If $\left(a_{1} m_{1}, a_{2} m_{1}, n\right) \in M_{1},\left(b_{1} m_{2}, b_{2} m_{2}, n\right) \in M_{1}$, and $\left(\left(a_{1}-a_{2}\right) m_{1}, n\right) \in M_{1}$, then $G^{\prime} / G^{\prime \prime}$ is finite.

Proof. The proof proceeds along similar lines to that of Theorem 2. We have

$$
\begin{aligned}
\frac{\partial r}{\partial x} & =\frac{\partial x^{a_{1}}}{\partial x}+x^{a_{1}} y^{b_{1}} \frac{\partial x^{a_{2}}}{\partial x} \\
& \equiv \alpha_{1}+\alpha_{2} z+\cdots+\alpha_{n} z^{n-1}\left(\bmod G^{\prime}\right)
\end{aligned}
$$

Let $f(z)=\alpha_{1}+\alpha_{2} z+\cdots+\alpha_{n} z^{n-1}$.

There are four cases to consider depending on the signs of $a_{1}$ and $a_{2}$. However in 
each case we obtain

$$
\left(1-z^{m_{1}}\right) f(z)=1-z^{a_{1} m_{1}}+z^{a_{1} m_{1}+b_{1} m_{2}}-z^{a_{1} m_{1}+b_{1} m_{2}+a_{2} m_{1}}
$$

Now, by Lemma 4 , det $A=\prod_{z^{n=1}} f(z)$ (where $A$ is the matrix equivalent to the matrix $A_{i}$ of Theorem 1). If $\operatorname{det} A=0$, then $f(w)=0$ for some $n$th root $w$ of unity, and if $\operatorname{det} A \neq 0$, then $G^{\prime} / G^{\prime \prime}$ is finite by Theorem 1 . Assume, for a contradiction, that $\operatorname{det} A=0$, so there exists $w$ such that $f(w)=0$, where $w^{n}=1$. Then $\left(1-w^{m_{1}}\right) f(w)=0$ and so, by (3),

$$
1-w^{a_{1} m_{1}}+w^{a_{1} m_{1}+b_{1} m_{2}}-w^{a_{1} m_{1}+b_{1} m_{2}+a_{2} m_{1}}=0 .
$$

Let $w^{a_{1} m_{1}}=p_{1}+i q_{1}, w^{a_{1} m_{1}+b_{1} m_{2}}=p_{2}+i q_{2}$, and $w^{a_{1} m_{1}+b_{1} m_{2}+a_{2} m_{1}}=p_{3}+i q_{3}$, where $p_{j}^{2}+q_{j}^{2}=1$ $(j=1,2,3)$, (where $i^{2}=-1$ ).

From (4) we have

$$
1-p_{1}+p_{2}-p_{3}=0 \Rightarrow p_{1}=1+p_{2}-p_{3}
$$

and $-q_{1}+q_{2}-q_{3}=0 \Rightarrow q_{1}=q_{2}-q_{3}$.

Now $p_{1}^{2}+q_{1}^{2}=1$, so $\left(1+p_{2}-p_{3}\right)^{2}+\left(q_{2}-q_{3}\right)^{2}=1$. Multiplying out, factorising, and squaring, we have

$$
\left(1+p_{2}\right)^{2}\left(1-p_{3}\right)^{2}=\left(1-p_{2}^{2}\right)\left(1-p_{3}^{2}\right)
$$

There are three cases to consider

(a) $p_{3}=1$, so $w^{a_{1} m_{1}+b_{1} m_{2}+a_{2} m_{1}}=1$, hence, by (4), $w^{a_{1} m_{1}}=w^{a_{1} m_{1}+b_{1} m_{2}} \Rightarrow w^{b_{1} m_{2}}=1$. Hence $w^{\left(a_{1}+a_{2}\right) m_{1}}=1$. Also $w^{\left(a_{1}+a_{2}\right) m_{1}+\left(b_{1}+b_{2}\right) m_{2}}=1$ (because $\left.r=z^{\left(a_{1}+a_{2}\right) m_{1}+\left(b_{1}+b_{2}\right) m_{2}}\left(\bmod G^{\prime}\right)\right)$, so $w^{b_{2} m_{2}}=1$.

(b) $p_{2}=-1$, so $w^{a_{1} m_{1}+b_{1} m_{2}}=-1$.

(c) $\left(1+p_{2}\right)\left(1-p_{3}\right)=\left(1-p_{2}\right)\left(1+p_{3}\right)$ i.e. $1+p_{2}-p_{3}-p_{2} p_{3}=1-p_{2}+p_{3}-p_{2} p_{3}$, hence $p_{2}=$ $p_{3}$. Thus, from (5), $p_{1}=1$, so $w^{a_{1 m_{1}}}=1$. By (4), $w^{b_{1} m_{2}}=w^{b_{1} m_{2}+a_{2} m_{1}}$, so $w^{a_{2 m_{1}}}=1$.

Assume that $n$ is odd. In case (b) $w^{a_{1} m_{1}+b_{1} m_{2}}=-1$, so this case has no solution (because $n$ is odd), so we need only consider cases (a) and (c). In case (a) $w^{b_{1} m_{2}}=1$ and $w^{b_{2} m_{2}}=1$, while in case (c) $w^{a_{1} m_{1}}=1$ and $w^{a_{2} m_{1}}=1$. So if $\left(a_{1} m_{1}, a_{2} m_{1}, n\right) \in M_{1}$ and $\left(b_{1} m_{2}, b_{2} m_{2}, n\right) \in M_{1}$, then $w^{m_{1}}=1$. Hence $f(w)=a_{1}+a_{2} w^{a_{1} m_{1}+b_{1} m_{2}}$. Now $w^{n}=1$, where $n$ is odd, and $a_{1}+a_{2} \neq 0$, so $f(w) \neq 0$ contradicting $\operatorname{det} A=0$. So $\operatorname{det} A \neq 0$ and $G^{\prime} / G^{\prime \prime}$ is finite by Theorem 1, proving (i).

Assume that $n$ is even. In case (b) $w^{a_{1} m_{1}+b_{1} m_{2}}=-1$, so, by (4), $w^{b_{1} m_{2}+a_{2} m_{1}}=-1$ and thus $w^{\left(a_{1}-a_{2}\right) m_{1}}=1$. In case (a) $w^{b_{1} m_{2}}=1$ and $w^{b_{2} m_{2}}=1$. In case (c) $w^{a_{1} m_{1}}=1$ and $w^{a_{2} m_{1}}=1$. So if $\left(a_{1} m_{1}, a_{2} m_{1}, n\right) \in M_{1},\left(b_{1} m_{2}, b_{2} m_{2}, n\right) \in M_{1}$, and $\left(\left(a_{1}-a_{2}\right) m_{1}, n\right) \in M_{1}$, then $w^{m_{1}}=1$. Thus $f(w)=a_{1}+a_{2} w^{a_{1} m_{1}+b_{1} m_{2}} . \quad f(w) \neq 0$, because $a_{1}+a_{2} \neq 0$ and $a_{1} \neq a_{2}$ (because $\left.\left(\left(a_{1}-a_{2}\right) m_{1}, n\right) \in M_{1}\right)$ contradicting $\operatorname{det} A=0$. So $\operatorname{det} A \neq 0$ and $G^{\prime} / G^{\prime \prime}$ is finite by Theorem 1 , proving (ii). 


\section{REFERENCES}

1. R. H. Fox, Free differential calculus 1, Ann. of Math. 57 (1953), 547-560.

2. J. R. Howse, An algorithm for the second derived factor group (Ph.D. thesis, University of Nottingham, 1984).

3. J. R. Howse and D. L. Johnson, An algorithm for the second derived factor group, in: Groups-St Andrews 1981, London Math. Soc. Lecture Note Series 71 (1982), 237-243.

4. T. MulR, A treatise on the theory of determinants (Dover Publications, New York, 1960).

Mid-Kent College

Chatham, ME5 9UQ

ENGLAND 